\title{
Biomimetic synthesis and characterization of semiconducting hybrid organic-inorganic composite materials based on polyaniline- polyethylene glycol-CdS system
}

\author{
A SINGH, N P SINGH and R A SINGH ${ }^{\dagger}$ \\ Department of Chemistry, Udai Pratap Autonomous Post Graduate College, Varanasi 221 001, India \\ ${ }^{\dagger}$ Department of Chemistry, Banaras Hindu University, Varanasi 221005 , India
}

MS received 2 September 2009; revised 12 January 2010

\begin{abstract}
Triple hybrid materials based on polyaniline-polyethylene glycol and cadmium sulphide have been prepared by the duffusion-limited biomimetic route and characterized by a number of spectroscopic, XRD, SEM, thermal and electrical measurements. These hybrid materials have been prepared by controlled precipitation of cadmium sulphide by passing $\mathrm{H}_{2} \mathrm{~S}$ gas and mixing the resultant colloid with the acidic solution of aniline. In situ polymerization of adsorbed anilinium ions on anionic surface of CdS resulted in hybrids. Water-soluble polyethylene glycol led to diffusion-limited growth of polyaniline and CdS resulting in a nanosized hybrid material as indicated by UV-visible spectra, X-ray diffraction (XRD) and scanning electron microscopy (SEM). AC impedance spectroscopic studies on binary and ternary nanocomposites of polyaniline with polyethylene glycol and cadmium sulphide separately and triple hybrid system have been reported. Equivalent circuits were determined and discussed in the light of contributions made from different sources such as grain, grain boundary and electrode.
\end{abstract}

Keywords. Polymers; chemical synthesis; differential scanning calorimetry; electrical properties.

\section{Introduction}

Conducting hybrid organic-inorganic nanomaterials have caught the attention of a number of research groups worldwide due to their unique electrical, optical, mechanical, thermal and magnetic properties (Nalwa 2001, 2002). The integration of organic semiconductors based on conducting polymers and conventional semiconductors such as metal sulphides and oxides on nanoscale has been envisaged as a useful process for developing novel optoelectronic devices that combine the best features of these two types of materials (Yang et al 2006). Such materials may play a crucial role not only in light-harvesting from UV to visible range due to absorptions from the two components but also facile transfer of photo-generated charge carriers through conjugated network of conducting polymers. These materials provide large interface for the dissociation of excitons and the transport of charge. Biomimetic approach (Castelvetro and Vita 2004) to materials chemistry has lead to new avenues for the synthesis of nanomaterials. It offers the possibility of controlling size, shape, crystal structure, orientation and organization of

\footnotetext{
*Author for correspondence (rasingh@bhu.ac.in)
}

nanomaterials (Klem et al 2005). Biomimetic synthesis of $\mathrm{CdS}$ nanocrystals in aqueous solution of pepsin at room temperature was reported recently (Mann 1997). Highly efficient resonant coupling of optical excitations have been found in the $J$-aggregates of cyanine dyes and crystalline semiconductor quantum dots grown by layer-bylayer self assembly (Zang et al 2007). Polyaniline-CdS system has been one of the best studied semiconducting polyaniline-metal sulphide system due to the commercial potential of both the components. CdS is a direct band gap semiconductor with band gap of $2.42 \mathrm{eV}$. It is used in photovoltaic and photosensitive devices such as photoresistors because its electrical resistance changes with the intensity of incident light. Mixed with $\mathrm{ZnS}$, it acts as a phosphor with a long-after-glow. Polyaniline is one of the most studied conducting polymers due to ease of synthesis, tunable electrical and optical properties dependent on various degrees of oxidation/reduction and proton doping of the polymer. One of the first reports on these composites appeared in 1991 not for the purpose of electronic properties but for the removal of cadmium ions with the help of sulfonated polyaniline (Dallas et al 1991). Although it was mentioned in the same paper that the controlled deposition of $\mathrm{CdS}$ on conducting polymers such as polyaniline may lead to the development of new photovoltaic elements, no attention was paid to these observations. The same authors had reported earlier the 
overgrowth of $\mathrm{CdS}$ on sulfonated polyaniline which yielded high conductivity and induced nucleation of $\mathrm{CdS}$ (Kallitsis et al 1989). Composite films of polyaniline and $\mathrm{CdS}$ were prepared by electrochemical polymerization of aniline in acidic solution containing suspended $\mathrm{CdS}$ particles (Yonema et al 1994). These films exhibited photoinduced electrochromism by illumination at open-circuit potential in presence of EDTA as electron donor. Electrochemical deposition of $\mathrm{CdS}$ nanoparticle-polyaniline composite films yielded a particle size of $30 \AA$ (Pethakar et al 1999). Films with good optoelectronic properties were prepared by controlling deposition parameters. Nanocomposites of polyaniline with $\mathrm{CdS}$ and $\mathrm{Cu}_{2} \mathrm{~S}$ of 1-2 nm sizes were prepared from their trifluoromethane sulfonates, co-dissolved in N-methyl pyrrolidone (NMP) (Godovsky et al 2001). Appreciable photovoltaic effect was observed in multilayer devices. $\mathrm{CdS}$ and $\mathrm{Cu}_{2} \mathrm{~S}$ were incorporated in polyaniline host matrix in which $\mathrm{CdS}$ was a $n$-type semiconductor and $\mathrm{Cu}_{2} \mathrm{~S}$ a $p$-type semiconductor (Chandrakanthi and Careem 2002). Photoluminescence studies on CdS quantum dots self-assembled in alternate layers with sulphonated polyaniline have been reported (Ma et al 2002). The intensity of luminescence increased with number of layers and heat treatment. Photovoltaic devices having varying concentrations of $\mathrm{CdS}$ and $\mathrm{Cu}_{2} \mathrm{~S}$ were fabricated and tested in dark and under illumination of $100 \mathrm{~mW} / \mathrm{cm}^{2}$. It was found that both short-circuit current $\left(I_{\mathrm{sc}}\right)$ and open-circuit potential $\left(V_{\mathrm{oc}}\right)$ increased with the $\mathrm{CdS}$ concentration. $\mathrm{CdS}$ coated with polyaniline was prepared by reverse micro-emulsion polymerization technique (Khiew et al 2004). Blue-shift in the UV-visible spectra of the composite was attributed to the quantumconfinement effect in the composite of average particle size of $17.8 \pm 2.7 \mathrm{~nm}$. Surfactant-stabilized nanoreactors provided cage-like effect that inhibited the excessive growth of coated nanoparticles. Dimethyl cadmium was used as an organometallic precursor in DMF (dimethyl formamide) to prepare polyaniline-CdS nanocomposites (Khanna et al 2004). A blue shift from $515 \mathrm{~nm}$ to $440 \mathrm{~nm}$ for $\mathrm{CdS}$ nanoparticles was reported. However, the proposed mechanism of interaction between zero-valent cadmium in cadmium dimethyl and nitrogen of polyaniline seems improbable for uniform distribution of $\mathrm{CdS}$ in polyaniline. Yellow and orange light emission from CdS nanoparticles embedded in polyvinyl alcohol was reported (Khanna et al 2005). Multi-modal particle size distribution of $\mathrm{CdS}$ nanoparticles led to broad patterns in the photoluminescence studies. Poly vinyl alcohol (PVA) was a particle growth protector for CdS in the composite synthesis of $\mathrm{HCl}$-doped polyaniline-CdS nanocomposites by use of organometallic precursor to prepare polyaniline blended with CdS (Khanna et al 2006). It was found that physical blending yielded composites with better electrical conductivities than ones prepared by in situ blending. It was also dependent on the $\mathrm{CdS}$ content and morphological changes produced by the inorganic component.
Optoelectronic properties of hybrid solar cells based on $\mathrm{CdS}$ nanorods and conjugated polymer were studied (Kang and Kim 2006). Improved efficiencies of solar cells were reported due to the organic-inorganic interface where bound electron-hole pairs could dissociate more effectively.

Recently, optical and electrical properties of selfassembled $\mathrm{CdS}$ nanorods-polyaniline composites were reported (Dutta et al 2007). Significant changes in electronic absorption spectra of CdS nanorods and polyaniline indicated strong interaction between them. Blue-shift in CdS from $530 \mathrm{~nm}$ to $475-432 \mathrm{~nm}$ in different samples was explained on the basis of quantum size effect. It was shown that synergistic effects between nanocrystalline $\mathrm{CdS}$ and polyaniline led to correlated barrier type conduction in these composites. CdSpolyaniline composites with improved thermal stability were reported. It was found that the electronic band of nanosized $\mathrm{CdS}$ found at $420 \mathrm{~nm}$ was blue-shifted due to quantum size effects but it overlapped with polyaniline.

It is evident from the above reports that polyaniline$\mathrm{CdS}$ system is an interesting hybrid material which has commercial potential. However, these composites are neither processable nor mechanically strong. Adding a third insulating polymer component will not only enhance the processability of the composites but will also work as a growth protector for nanomaterials. The present work reports the biomimetic synthesis of mono-, bi- and tri- component systems based on polyanilinepolyethylene glycol-CdS on which detailed a.c. impedance spectroscopic studies have been made. The samples have been characterized by FTIR, UV-visible spectroscopy, XRD, differential scanning calorimetry (DSC) and SEM. Grain, grain-boundary and electrode contributions to the total a.c. impedance have been separated using complex non-linear least square (CNLS) software. Temperature dependence of electrical properties has been studied to learn about the transport mechanism in these hybrid materials. It is expected that these data will help in designing optoelectronic devices based on a new class of semi conducting organic-inorganic hybrid materials.

\section{Experimental}

\subsection{Material and methods}

Aniline (Merck, A.R. grade) was distilled twice at normal pressure before use. Polyethylene glycol 6000 (Average molecular weight 7000-9000) (LOBA chemicals, A.R. grade), cadmium nitrate (Qualigen) and ammonium per disulphate (Merck) were used as received. Triple distilled water, obtained from a quartz double distillation unit, was used for the synthesis. 


\subsection{Synthesis}

Due to toxicity of cadmium, utmost care was taken while handing it. Polyaniline was prepared by using standard procedures (Trivedi 1997). The composites with polyethylene glycol were prepared by the same procedure using $1 \mathrm{~g}$ polyethylene glycol dissolved in water.

\subsection{Polyaniline and cadmium sulphide composite}

Aniline, $3 \mathrm{~g}(0.03 \mathrm{~mol})$ was dissolved in $60 \mathrm{ml} 2 \mathrm{~N} \mathrm{HCl}$ and kept in ice bath. $6 \mathrm{~g}(0.025 \mathrm{~mol})$ cadmium nitrate was dissolved in $50 \mathrm{ml} 2 \mathrm{~N} \mathrm{HCl}$ into which $\mathrm{H}_{2} \mathrm{~S}$ was passed for $10 \mathrm{~min}$. A yellow coloured colloidal cadmium sulphide was obtained which was cooled to a temperature $<5^{\circ} \mathrm{C}$. A pre-cooled solution of $9 \mathrm{~g}(0.04 \mathrm{~mol})$ of ammonium per disulphate dissolved in $60 \mathrm{ml}$ water was added slowly by burette into the aniline hydrochloride and cadmium sulphide mixture with continuous stirring. A green coloured precipitate formed indicating the formation of polyaniline. The reaction was allowed to proceed for $50 \mathrm{~min}$, and then the mixture was kept in freezer overnight. After polymerization, a bluish green colour precipitate was obtained. The precipitate was filtered and washed with $2 \mathrm{~N} \mathrm{HCl}$ and thrice by triple distilled water in order to remove unreacted reagents. The precipitate was dried in an oven at $60-70^{\circ} \mathrm{C}$ and then kept over anhydrous $\mathrm{CaCl}_{2}$ in a desiccator. The yield was $98 \%$ (w/w ratio). The efforts to prepare $\mathrm{CdS}$-polyaniline composite from a mixture of cadmium nitrate and aniline hydrochloride and passing $\mathrm{H}_{2} \mathrm{~S}$ and adding ammonium per disulphate simultaneously yielded a very small amount of composite due to formation of yellow elemental sulfur on reduction of hydrogen sulphide by ammonium per disulphate. It was tested by passing $\mathrm{H}_{2} \mathrm{~S}$ in ammonium per disulphate solution.

\subsection{Polyaniline-polyethylene glycol-cadmium sulphide composite}

Aniline, $3 \mathrm{~g}(0.03 \mathrm{~mol})$ was dissolved in $60 \mathrm{ml} 2 \mathrm{~N} \mathrm{HCl}$ and kept in ice bath, and then mixed thoroughly with $1 \mathrm{~g}$ polyethylene glycol dissolved in $25 \mathrm{ml}$ water. $6 \mathrm{~g}$ $(0.025 \mathrm{~mol})$ cadmium nitrate was dissolved in $50 \mathrm{ml} 2 \mathrm{~N}$ $\mathrm{HCl}$ and $\mathrm{H}_{2} \mathrm{~S}$ passed into it for $10 \mathrm{~min}$. A yellow coloured colloidal cadmium sulphide was obtained, which was mixed thoroughly in aniline- $\mathrm{HCl}$ and polyethylene glycol mixture and cooled below $5^{\circ} \mathrm{C}$ in an ice bath. Ammonium per disulphate $9 \mathrm{~g}(0.04 \mathrm{~mol})$ dissolved in $60 \mathrm{ml}$ water was added slowly in an hour along with continuous stirring. The green colour precipitate was kept in freezer overnight, filtered, washed and dried. The yield was $84 \%$ (w/w).

\section{Measurements}

FT-IR spectra of the samples were recorded using Varian $3100 \mathrm{FT}-\mathrm{IR}$ Excalibur series in the range $400-4000 \mathrm{~cm}^{-1}$ in $\mathrm{KBr}$ medium. UV-visible spectra was obtained by UV1700 Pharma Spec. Simadzu corp. in the range 300$1100 \mathrm{~nm}$ in nujol medium. Differential scanning calorimetry measurements were made using a TC 15 TA Controller Mettler Tole Do. instrument. The powder X-ray diffraction of the polyaniline, polyethylene glycol and CdS composites were obtained from a Rigaku (ModelRINT-2000; Japan) X-ray diffractometer using $\mathrm{CuK} \alpha$ radiation $(1.542 \AA)$ in the $2 \theta$ range $5-60^{\circ} \mathrm{C}$. Scanning electron microscopic studies were conducted using Leica Cambridge 440 microscope. AC impedance/conductance in the frequency range of $40 \mathrm{~Hz}-100 \mathrm{KHz}$ and temperature range of $308-348 \mathrm{~K}$ were made using a LCZ meter (Keithley 3330) and data were simulated using EG\&G PARC USA CNLS software to determine equivalent circuits. The basic accuracy of the LCZ meter used for measurement was $0 \cdot 1 \%$ for the impedances in the range of $0.1 \mathrm{~m} \Omega$ to $19.999 \mathrm{M} \Omega$; capacitances in the range of $0.001 \mathrm{pF}$ to $199.99 \mathrm{mF}$ and for conductance in the range of $0.001 \mu \mathrm{S}$ to $199.99 \mathrm{~S}$. The relaxation times were evaluated from the products of $R$ and $C$ obtained from the equivalent circuit analysis. The thermal energy of activation was determined from the Arrhenius plots between logarithm of specific conductivity $(\log \sigma)$ vs inverse temperature $\left(T^{-1}\right)$.

\section{Results and discussion}

\subsection{Synthesis}

The mechanism of formation of polyaniline-polyethylene glycol-cadmium sulphide hybrid: The formation of nanosized triple hybrid materials based on polyanilinepolyethylene glycol-CdS is illustrated in figure 1. Polyethylene glycol sheets act as a biomimetic synthesis aid by separating anilinium hydrochloride molecules which remain isolated after the reaction with ammonium per disulphate leading to smaller (nano-sized) particles of polyaniline (figure 1a). On addition of $\mathrm{CdS}$ anilinium ions are adsorbed on it and these particles remain isolated in the polyethylene glycol matrix. On addition of the oxidant, polymerization of aniline occurs on the CdS templates forming a core-shell nanostructure as supported by the uniform bluish-green colour and absence of any yellow CdS and also by SEM studies discussed below (figure 1b).

\subsection{FTIR spectra}

The FT-IR spectra of the hybrids and pure samples are shown in figure 2 . A very good agreement between these 
spectra and those reported by Khiew (Khiew et al 2004) for polyaniline-cadmium sulphide nanomaterials prepared in emulsion has been found indicating that biomimetic synthesis leads to hybrid nano-materials based on these systems. The addition of water soluble third component i.e. poly ethylene glycol as a biomimetic synthesis agent not only controls the particle size but also enhances the processability of hybrid materials. For example, characteristic bands for the conducting polyaniline form were observed at 3444, 3226, 2924, 2856, 1566, 1482, 1300, 1243,800 and $703 \mathrm{~cm}^{-1}$ according to literature (Kang et al 1998; Khanna et al 2006). The bands at $1482 \mathrm{~cm}^{-1}$ were found to undergo a red-shift to $1466 \mathrm{~cm}^{-1}$ in presence of CdS along with similar shifts from 1144 to $1118 \mathrm{~cm}^{-1}$ and 703 to $672 \mathrm{~cm}^{-1}$. Nitrogen is associated with these bands and red shifts indicate weakening of bonds by interaction with metal ion centre. Similar interactions between polyaniline and CdS have been proposed earlier as well, but the nature of these interactions seems to be particulate rather than at the molecular level as proposed for CdS-SPAN system (Ma et al 2002). The interaction of cationic anilinium ions with anionic $\mathrm{CdS}$ colloidal particle may be responsible for bringing $\mathrm{CdS}$ particles close to the nitrogen sites which lead to weak interactions between the conducting polyaniline shell with
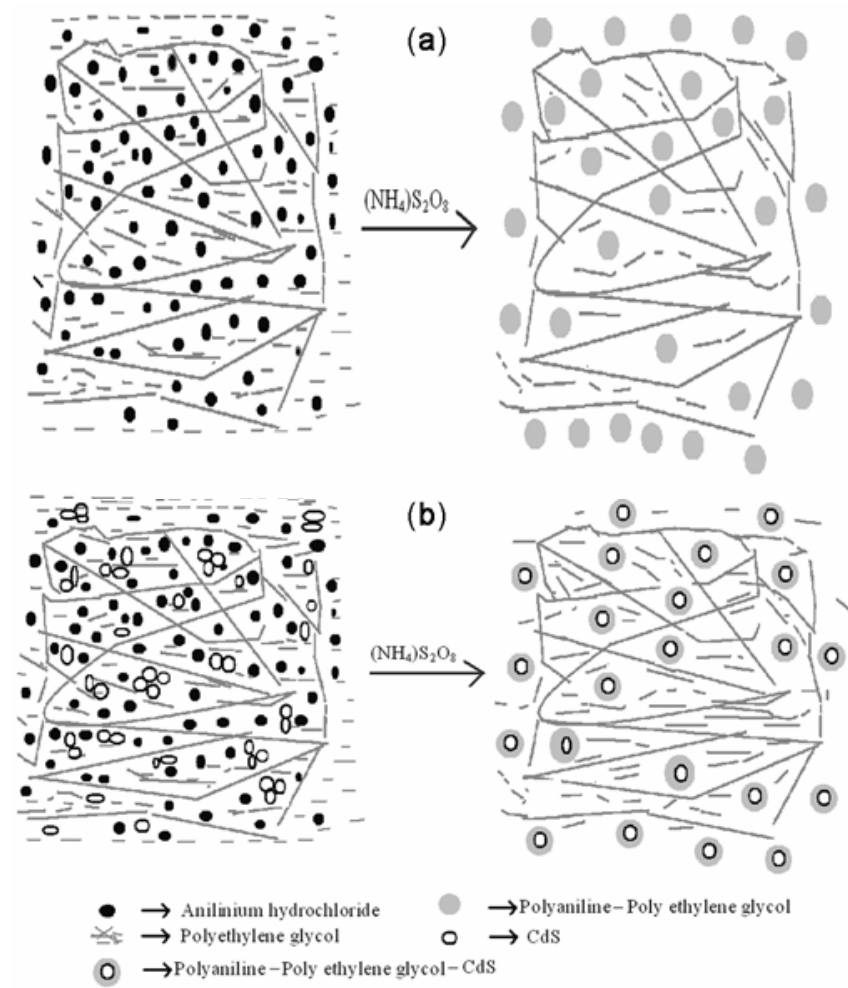

Figure 1. Proposed mechanism for formation of polyaniline, polyethylene glycol and cadmium sulphide particle. a. Polyaniline-polyethylene glycol and b. polyaniline-polyethylene glycol-cadmium sulphide. non-conducting CdS core. Such molecular interactions have been proposed for the formation of rod-like CdSpolyaniline nanostructure (Dutta et al 2007).

\section{$4.3 \quad$ UV-Visible spectra}

UV-Visible spectra are helpful not only to identify the nanomaterials but also the extent of conjugation in conducting polymers (Nalwa 2001). The spectra for various samples prepared by biomimetic route are given figure 3 . Pure polyaniline bands at $868 \mathrm{~nm}$ indicate high degree of conjugation and a high conductivity of $0.44 \mathrm{Scm}^{-1}$ at room temperature. The UV-Visible spectra of polyaniline-polyethylene glycol-CdS system exhibit exactly similar behaviour as polyaniline- $\mathrm{SiO}_{2}$ core-shell nanostructure reported recently (Jang et al 2006). Thus a formation of core-shell nanostructured material based on polyaniline and CdS could be described by the mechanism proposed by these authors. An additional proof of the existence of $\mathrm{CdS}$ nanoparticles came from the blue-shift of particulate CdS visible band at $\sim 500 \mathrm{~nm}$ to around $430 \mathrm{~nm}$ due to size quantization effects

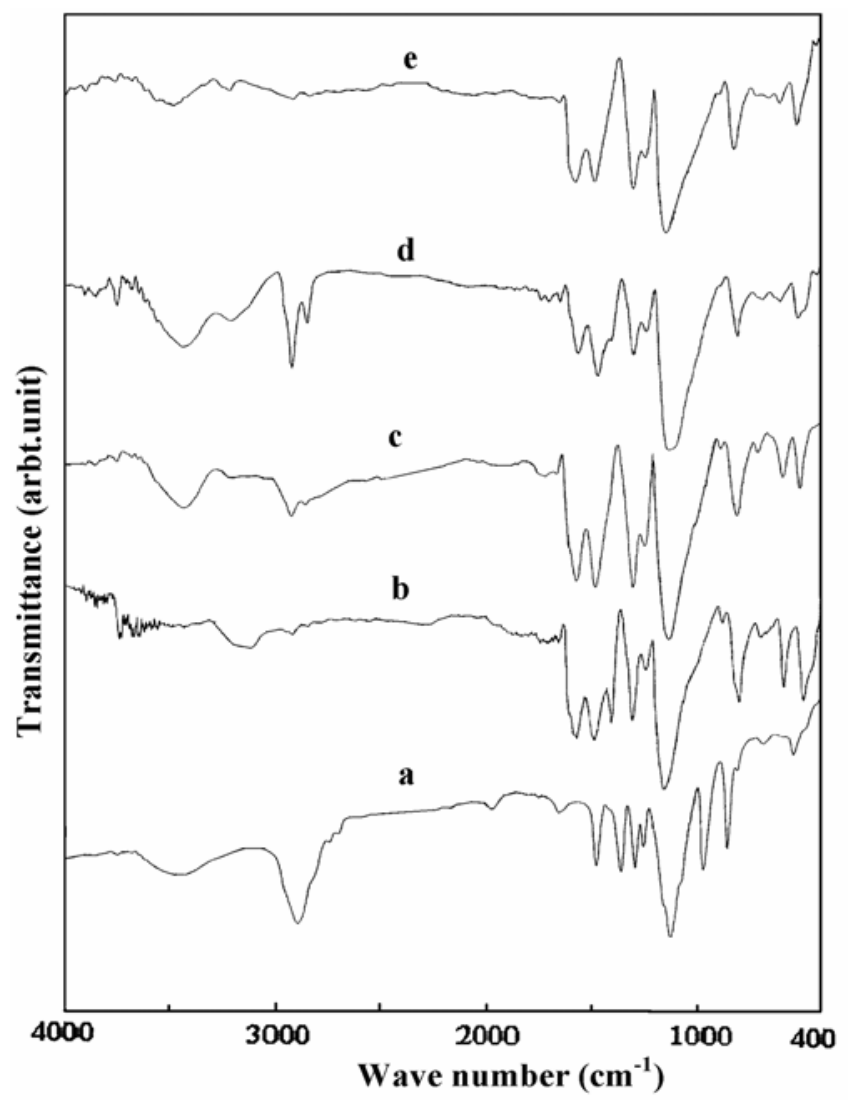

Figure 2. Infra-red spectra of (a) polyethylene glycol, (b) polyaniline, (c) polyaniline-polyethylene glycol, (d) polyaniline-cadmium sulphide and (e) polyaniline-polyethylene glycol-cadmium sulphide. 
(Singh et al 2007). It may be pointed out that the band due to nano-sized $\mathrm{CdS}$ at $430 \mathrm{~nm}$ overlapped with the excitonic transition of polyaniline around $420 \mathrm{~nm}$ and the band around $500 \mathrm{~nm}$ in particulate $\mathrm{CdS}$ was absent in the composite. There were no yellow coloured particles of $\mathrm{CdS}$ present in the preparations containing polyaniline

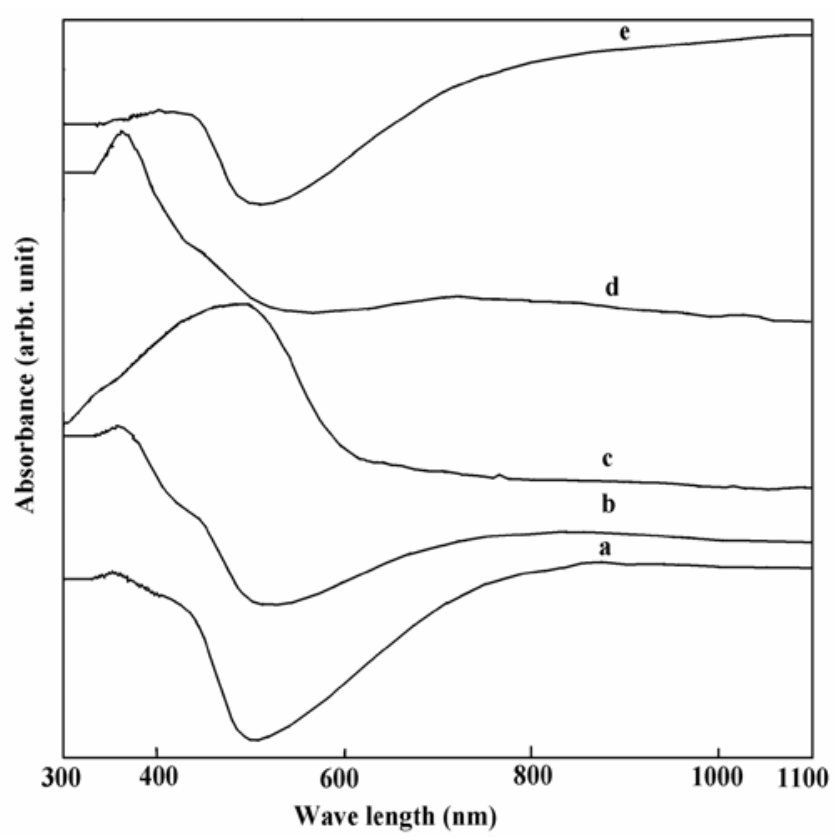

Figure 3. UV-Visible spectra of (a) polyaniline, (b) polyaniline-polyethylene glycol, (c) cadmium sulphide, (d) polyaniline-cadmium sulphide and (e) polyaniline-polyethylene glycol-cadmium sulphide.

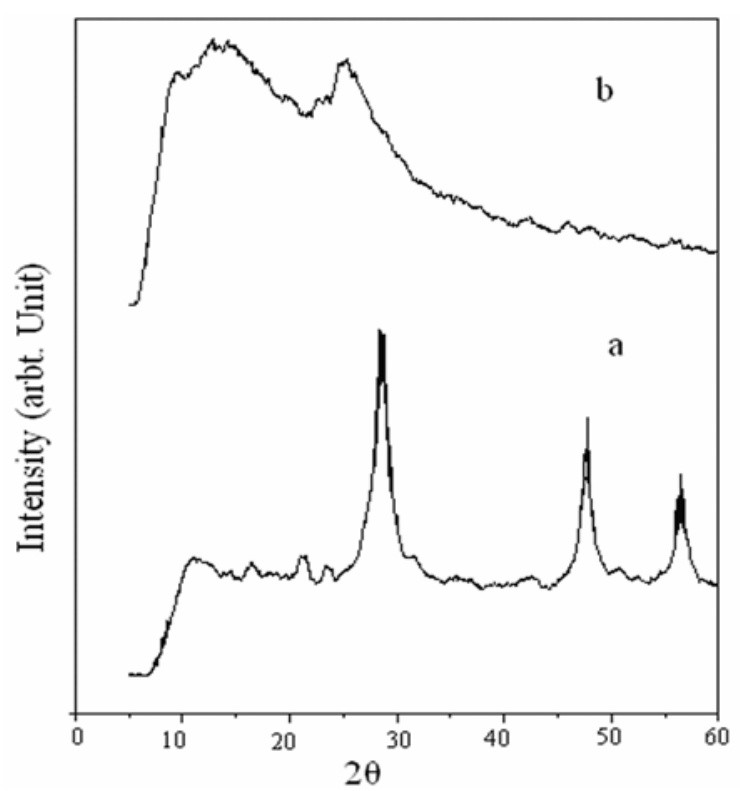

Figure 4. XRD spectra of (a) cadmium sulphide and (b) polyaniline-polyethylene glycol-cadmium sulphide. and $\mathrm{CdS}$ indicating good coating of $\mathrm{CdS}$ nanoparticles by polyaniline. The colour of the composite material was similar to pure polyaniline and one-to-one matching in UV-Visible peaks were observed. The broadbands between 700 and $1100 \mathrm{~nm}$ indicate extensive conjugation which in turn yielded highly conducting composites.

\section{$4.4 \quad$ X-ray diffraction}

X-ray diffraction (XRD) patterns of different samples are given in figure 4 . It is evident from the figure that pure CdS had sharp peaks indicating its crystalline nature but the composite had broad diffuse peaks due to its amorphous behaviour. In cadmium sulphide, three sharp peaks at $d$-values of $3.1403,1.9013$ and $3.8504 \AA$ with respective diffraction angles of $2 \theta$ at $28^{\circ} \mathrm{C}, 48^{\circ} \mathrm{C}$ and $56^{\circ} \mathrm{C}$ were obtained. These values are quite different from the crystal structure data of this compound reported in XRD data files indicating a different form of the material prepared by our method probably due to its nanocrys-

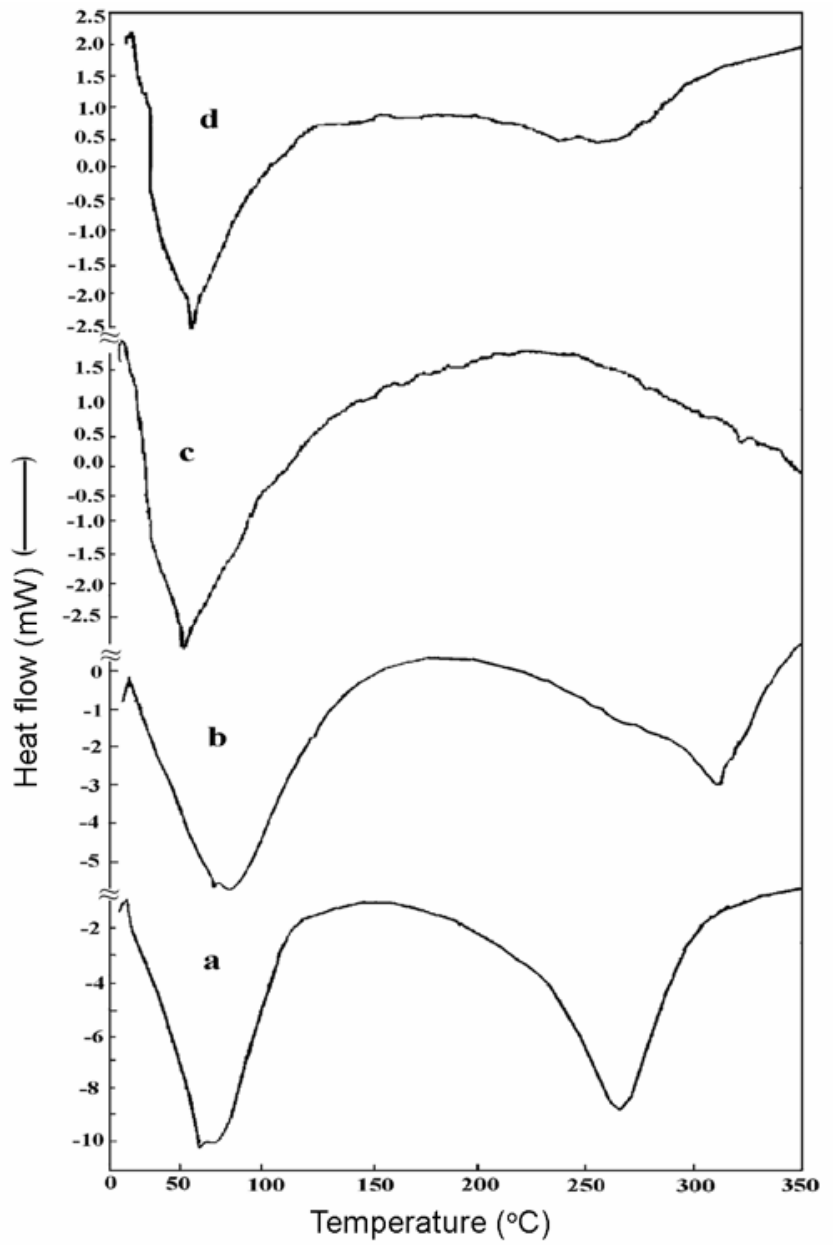

Figure 5. DSC thermograms of (a) polyaniline, (b) polyaniline-polyethylene glycol, (c) polyaniline-cadmium sulphide and (d) polyaniline-polyethylene glycol-cadmium sulphide. 
talline nature. X-ray diffraction of polyanilinepolyethylene glycol-CdS composite showed broad peaks at $7.2051 \AA$ and $3.8504 \AA$ interplanar spacing with corresponding $2 \theta$ values of $13^{\circ} \mathrm{C}$ and ca $24^{\circ} \mathrm{C}$. These data are analogous to those reported recently for polyaniline- $\mathrm{CdS}$ polymer nanocomposites (Chandrakanthi and Careem 2002). The crystalline domain size, $L$, was estimated using the Scherrer formula (Pouget et al 1991).

$$
L=\frac{0 \cdot 9 \lambda}{\Delta(2 \theta) \cos \theta}
$$

where $L$ is the size of the particle, $\lambda=\mathrm{X}$-ray wave length, $\Delta(2 \theta)=$ full width at half-maximum (fwhm).

The average particle size $(L)$ of polyaniline-polyethylene glycol-CdS was $40 \mathrm{~nm}$ and for $\mathrm{CdS}$ it was $22 \mathrm{~nm}$. These data not only confirm the formation of nanocomposites but also the increase in particle size supported the intimate coating mechanism proposed in figure 1 .

\subsection{Differential scanning calorimetry measurements}

Differential scanning calorimetry (DSC) of some composite samples as shown in figure 5. These figures also match with the recent measurements on polyaniline system indicating the formation of similar materials (Gangopadhyay et al 2001). Two endothermic peaks were observed for pure polyaniline; one having a peak temperature at $70.7^{\circ} \mathrm{C}$ and integral heat of $4528 \mathrm{~mJ}$ and normalized heat of $427 \mathrm{Jg}^{-1}$ and the other with a peak at $264 \cdot 3{ }^{\circ} \mathrm{C}$ with integral heat of $4277 \mathrm{~mJ}$ and normalized heat of $404 \mathrm{Jg}^{-1}$. When polyaniline was prepared in presence of polyethylene glycol, the first endothermic peak at $71 \cdot 1^{\circ} \mathrm{C}$ showed a decrease in the heat from 4528 to $3304 \mathrm{~mJ}$. Similarly, the second peak showed a decrease from 4277 to $2618 \mathrm{~mJ}$, indicating the presence of another component facilitated the melting of the polyaniline. A drastic decrease in the first integral heat from 4528 to $248 \mathrm{~mJ}$ was seen for polyaniline-CdS system and to $222 \mathrm{~mJ}$ for polyaniline-polyethylene glycol-CdS systems, respectively. The second endothermic peak was eliminated in the hybrid system. These thermal data indicate the softening of polyaniline on addition of polyethylene glycol and CdS thus enhancing their thermoplasticity and hence the thermoprocessing.

\subsection{Scanning electron microscopy studies}

Scanning electron microscopic image of the triple hybrid is given in figure 6 . It was proposed in figure 1 that the colloidal CdS particles had a nucleation effect on the polymerization of aniline leading to homogeneous coating of $\mathrm{CdS}$ with polyaniline. It was further pointed out that $\mathrm{CdS}$ had strong effect on morphology of polyaniline from fibrous to particulate depending on the polyaniline con- tent. The SEM images not only support these propositions but also the particle size estimates from XRD. These microstructures also support our equivalent circuit analysis discussed below in terms of RC circuit from grain contribution with single time constants. The average diameters for the polyaniline-CdS nanocomposite particles were estimated to be ca $17.8 \mathrm{~nm}$ with a standard deviation of $2.7 \mathrm{~nm}$ using TEM (Khiew et al 2004). These values are approximately double for the triple hybrid prepared by the biomimetic route.

\subsection{AC impedance studies}

Electrical properties of composites are highly dependent on synthetic conditions. AC measurements not only avoid the polarization effects but also lead to separation of various contributions to total impedance or conductance. Highly conducting polyaniline-CdS, were prepared, having room temperature conductivities in the range of $1.85 \times 10^{-2}$ to $2.53 \times 10^{-1} \mathrm{Scm}^{-1}$ depending on the amount of aniline taken from $1.5-5 \mathrm{~g}$ (keeping the amount of $\mathrm{Cd}\left(\mathrm{NO}_{3}\right)_{2}$ fixed at $6 \mathrm{~g}$ ). Addition of

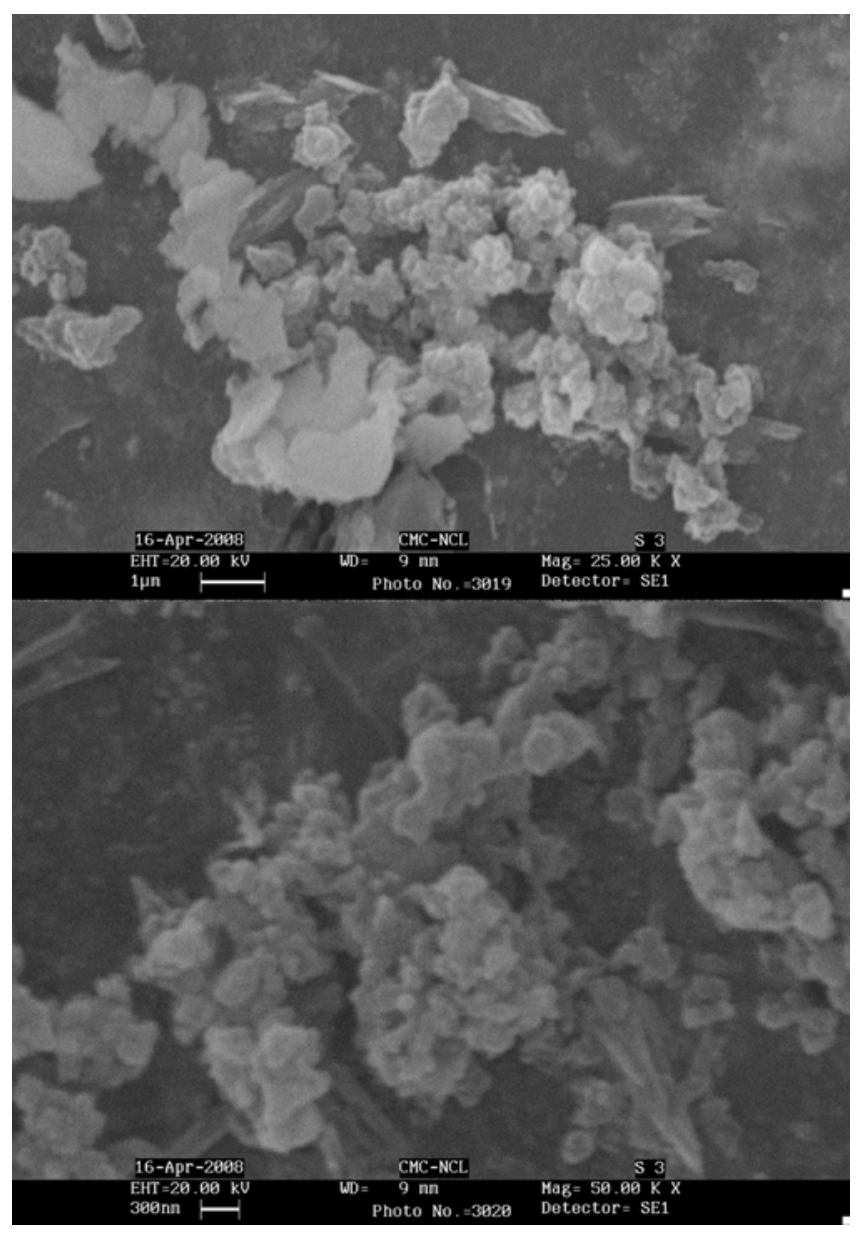

Figure 6. SEM micrographs of polyaniline-polyethylene glycol-cadmium sulphide 

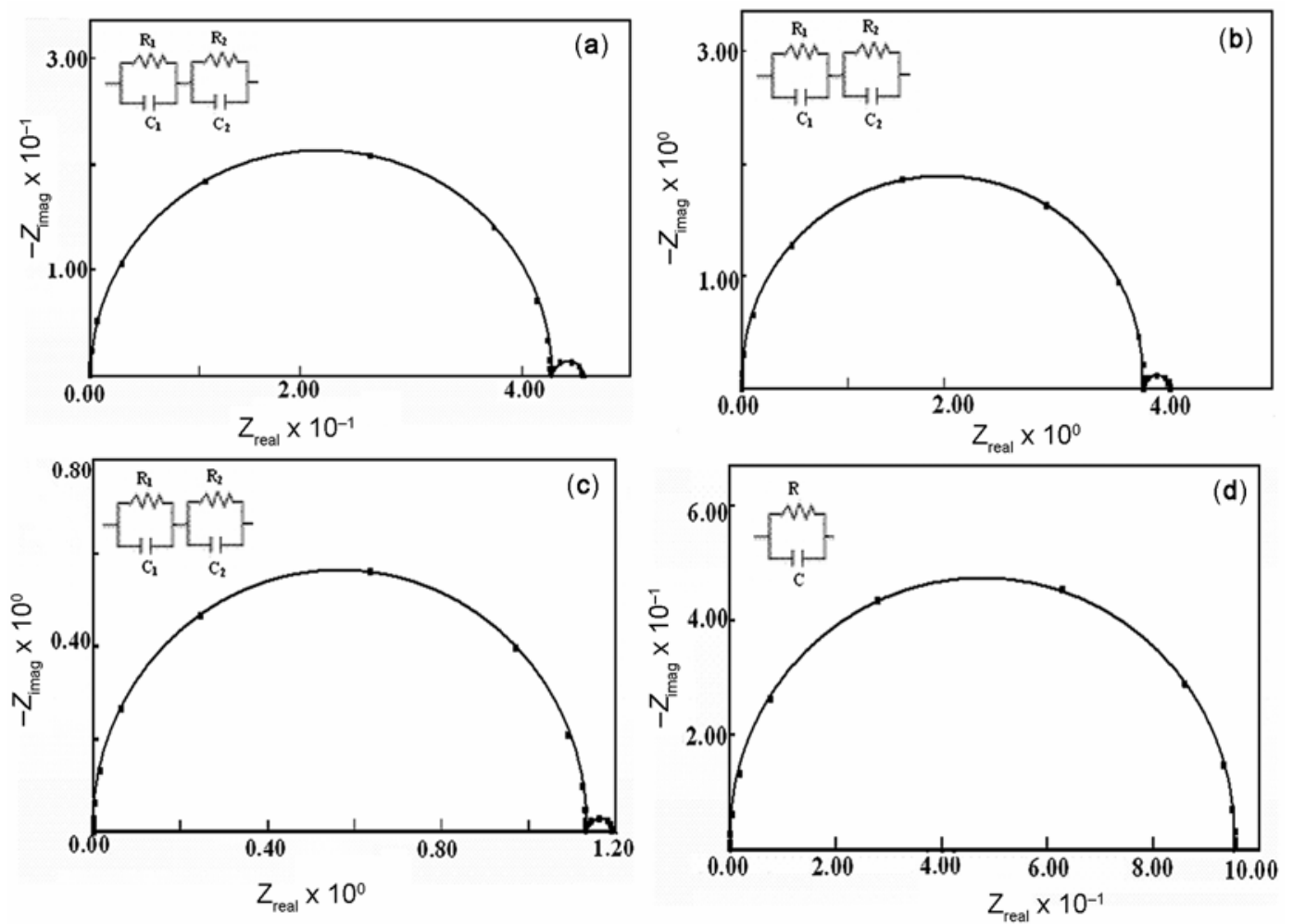

Figure 7. Complex impedance plots (a) polyaniline, (b) polyaniline-polyethylene glycol, (c) polyaniline-cadmium sulphide and (d) polyaniline-polyethylene glycol-cadmium sulphide.

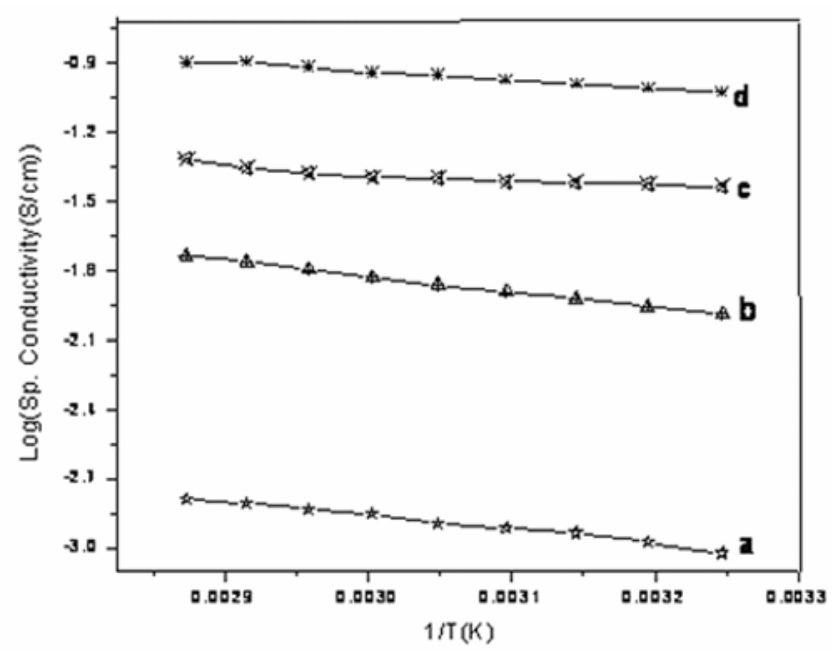

Figure 8. Arrhenius plots for (a) polyaniline-cadmium sulphide, (b) polyaniline, (c) polyaniline-polyethylene glycol and (d) polyaniline-polyethylene glycol-cadmium sulphide.

polyethylene glycol in the range $0.5-1.6 \mathrm{~g}$ changes the conductivity from $1.34 \times 10^{-2}$ to $2.06 \times 10^{-1} \mathrm{Scm}^{-1}$. Addition of CdS prepared from aqueous solution to polyaniline gives slightly higher value of conductivity from $2.50 \times 10^{-1}$ to $3.17 \times 10^{-1} \mathrm{Scm}^{-1}$ indicating no effect of presence of acidic solution $(2 \mathrm{~N})$ on the formation and stability of CdS colloids. Similarly a composite with CdS plus $\mathrm{ZnS}$ yielded a conductivity of $3.32 \times 10^{-1}$ $\mathrm{Scm}^{-1}$. These results indicate that highly conducting, reproducible hybrid materials can be prepared by biomimetic route. The AC impedance measurements for a representative sample; polyaniline- $\mathrm{CdS}$, made at various frequencies are given in table 1 and its simulated values for the determination of equivalent circuits in table 2 . These data are a representative set given with the purpose of illustrating the analysis of equivalent circuits. Impedance plots for different samples given in figure 7 show distinct changes in the nature of arcs. Pure polyaniline and its composite with polyethylene glycol have two similar arcs and circuit elements indicating good match between these samples. However, pure cadmium sulphide had three arcs indicating significant contributions from grain, grain-boundary and electrode. It is interesting to note that the triple hybrid of polyaniline-polyethylene glycol-CdS had only one arc indicating some sort of synergistic effect reported earlier (Dutta et al 2007). The circuit elements for the various samples are reported in table 3. Grain and electrode contributions could easily be separated but grain-boundary contributions could not be obtained from both the impedance and modulus plots except for CdS-polyethylene glycol system. The 
Table 1. Measured a.c. impedance data of polyaniline-cadmium sulfide composite* using ammonium per disulphate as oxidizing agent.

\begin{tabular}{|c|c|c|c|c|c|c|c|}
\hline $\begin{array}{l}v \\
\text { (frequency, } \\
\mathrm{Hz} \text { ) }\end{array}$ & $\begin{array}{c}\mathrm{Cp} \times 10^{-8} \\
\text { (capacitance, } \\
\text { Farad) }\end{array}$ & $\begin{array}{c}Z_{\|} \\
\text {(impedance, } \\
\text { Ohm) }\end{array}$ & $\begin{array}{c}Q \\
\text { (quality } \\
\text { factor) }\end{array}$ & $\begin{array}{c}D \\
\text { (dissipation } \\
\text { factor) }\end{array}$ & $\begin{array}{c}\sigma \times 10^{-2} \\
\text { (conductivity } \\
\mathrm{S} / \mathrm{cm} \text { ) }\end{array}$ & $\begin{array}{c}X \\
\text { (reactance, } \\
\text { Ohm) }\end{array}$ & $\begin{array}{c}\theta \\
\text { (phase angle) }\end{array}$ \\
\hline $4.00 \times 10^{1}$ & $20 \cdot 00$ & $1 \cdot 2421$ & $0 \cdot 0000$ & $\mathrm{OF}$ & $5 \cdot 554$ & $0 \cdot 0000$ & $0 \cdot 00$ \\
\hline $6.00 \times 10^{1}$ & $20 \cdot 00$ & 1.2370 & $0 \cdot 0000$ & $\mathrm{OF}$ & 5.672 & 0.0000 & 0.00 \\
\hline $8.00 \times 10^{1}$ & $20 \cdot 00$ & $1 \cdot 2142$ & $0 \cdot 0000$ & OF & $5 \cdot 716$ & 0.0000 & 0.00 \\
\hline $1.00 \times 10^{2}$ & $20 \cdot 00$ & 1.2118 & $0 \cdot 0000$ & OF & 5.783 & 0.0000 & $0 \cdot 00$ \\
\hline $2.00 \times 10^{2}$ & $10 \cdot 00$ & 1.1797 & 0.0001 & 15543 & $5 \cdot 896$ & 0.0001 & $0 \cdot 00$ \\
\hline $3.00 \times 10^{2}$ & $10 \cdot 00$ & $1 \cdot 1664$ & 0.0001 & 10255 & 5.966 & 0.0001 & $0 \cdot 01$ \\
\hline $4.00 \times 10^{2}$ & $10 \cdot 00$ & $1 \cdot 1650$ & $0 \cdot 0002$ & 6828 & 5.998 & $0 \cdot 0002$ & $0 \cdot 01$ \\
\hline $5.00 \times 10^{2}$ & $10 \cdot 00$ & $1 \cdot 1561$ & $0 \cdot 0002$ & 5495 & $6 \cdot 021$ & 0.0002 & $0 \cdot 01$ \\
\hline $6.00 \times 10^{2}$ & $10 \cdot 00$ & $1 \cdot 1552$ & $0 \cdot 0002$ & 4112 & $6 \cdot 047$ & 0.0003 & $0 \cdot 01$ \\
\hline $7.00 \times 10^{2}$ & $6 \cdot 00$ & $1 \cdot 1468$ & $0 \cdot 0003$ & 3644 & $6 \cdot 066$ & 0.0003 & 0.02 \\
\hline $8.00 \times 10^{2}$ & $6 \cdot 00$ & $1 \cdot 1462$ & $0 \cdot 0003$ & 3470 & $6 \cdot 096$ & 0.0004 & 0.02 \\
\hline $1.00 \times 10^{3}$ & 5.00 & $1 \cdot 1390$ & 0.0004 & 2679 & $6 \cdot 115$ & 0.0004 & 0.02 \\
\hline $2.00 \times 10^{3}$ & $5 \cdot 00$ & $1 \cdot 1380$ & 0.0007 & 1422 & $6 \cdot 136$ & 0.0008 & 0.04 \\
\hline $3.00 \times 10^{3}$ & 5.00 & $1 \cdot 1320$ & $0 \cdot 001$ & 970 & $6 \cdot 151$ & 0.0012 & 0.06 \\
\hline $5.00 \times 10^{3}$ & 5.00 & $1 \cdot 1317$ & $0 \cdot 0017$ & 600 & $6 \cdot 168$ & 0.0019 & $0 \cdot 1$ \\
\hline $7.00 \times 10^{3}$ & $5 \cdot 00$ & $1 \cdot 1264$ & 0.0022 & 450 & $6 \cdot 182$ & 0.0025 & $0 \cdot 13$ \\
\hline $9.00 \times 10^{3}$ & $4 \cdot 40$ & $1 \cdot 1260$ & 0.0028 & 360 & $6 \cdot 200$ & 0.0031 & $0 \cdot 16$ \\
\hline $1.00 \times 10^{4}$ & $4 \cdot 40$ & $1 \cdot 1209$ & 0.0031 & 324 & $6 \cdot 211$ & 0.0035 & $0 \cdot 18$ \\
\hline $2.00 \times 10^{4}$ & $4 \cdot 00$ & $1 \cdot 1212$ & 0.0056 & 178 & $6 \cdot 228$ & 0.0063 & $0 \cdot 32$ \\
\hline $4.00 \times 10^{4}$ & $3 \cdot 30$ & $1 \cdot 1166$ & 0.0095 & 103 & $6 \cdot 234$ & 0.0105 & $0 \cdot 54$ \\
\hline $6.00 \times 10^{4}$ & $3 \cdot 10$ & $1 \cdot 1169$ & $0 \cdot 0129$ & 77 & $6 \cdot 246$ & 0.0145 & $0 \cdot 75$ \\
\hline $8.00 \times 10^{4}$ & $2 \cdot 91$ & $1 \cdot 1125$ & $0 \cdot 0161$ & 62 & $6 \cdot 259$ & 0.0179 & 0.92 \\
\hline $1.00 \times 10^{5}$ & $2 \cdot 74$ & $1 \cdot 1120$ & $0 \cdot 0191$ & 53 & $6 \cdot 272$ & 0.0212 & 1.09 \\
\hline
\end{tabular}

*Weight of aniline $=3.0 \mathrm{~g}$, weight of cadmium sulphide $=2.70 \mathrm{~g}$, weight of ammonium per disulphate $=9 \cdot 0 \mathrm{~g}$. OF $\rightarrow$ overflow.
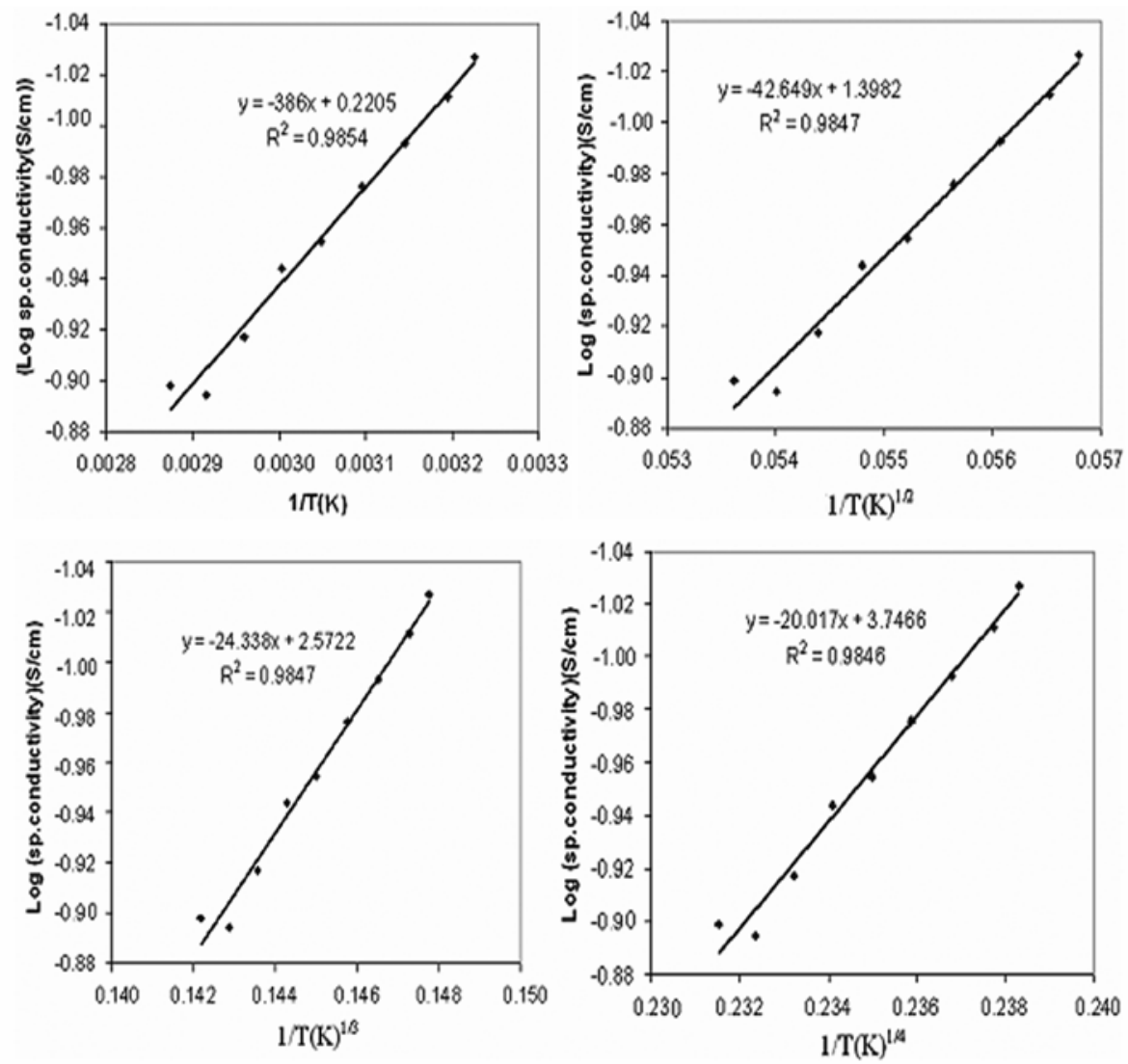

Figure 9. Regression analysis graph of polyaniline-polyethylene glycol-cadmium sulphide. 
Table 2. Simulated AC impedance data of polyaniline-cadmium sulfide composite using ammonium per disulphate as oxidizing agent.

\begin{tabular}{|c|c|c|c|c|c|c|}
\hline $\begin{array}{l}v \\
\text { (frequency, } \\
\mathrm{Hz} \text { ) }\end{array}$ & $\begin{array}{c}C \\
\text { (capacitance, } \\
\text { Farad) }\end{array}$ & $\begin{array}{c}Z_{\|} \\
\text {(impedance, } \\
\text { Ohm) }\end{array}$ & $\begin{array}{c}D \\
\text { (dissipation, } \\
\text { factor) }\end{array}$ & $\begin{array}{c}\sigma \times 10^{-2} \\
\text { (conductivity } \\
\mathrm{S} / \mathrm{cm} \text { ) }\end{array}$ & Permittivity & $\begin{array}{c}\theta \\
\text { (phase angle) }\end{array}$ \\
\hline $1.00 \times 10^{-3}$ & $1.363 \times 10^{-5}$ & $1.191 \times 10^{0}$ & $9.811 \times 10^{6}$ & $5 \cdot 851$ & $1.073 \times 10^{7}$ & $5.84 \times 10^{-6}$ \\
\hline $4.64 \times 10^{-3}$ & $1.363 \times 10^{-5}$ & $1.191 \times 10^{0}$ & $2.113 \times 10^{6}$ & $5 \cdot 851$ & $1.073 \times 10^{7}$ & $2.71 \times 10^{-5}$ \\
\hline $2 \cdot 15 \times 10^{-2}$ & $1.363 \times 10^{-5}$ & $1.191 \times 10^{0}$ & $4.553 \times 10^{5}$ & $5 \cdot 851$ & $1.073 \times 10^{7}$ & $1.26 \times 10^{-4}$ \\
\hline $1.00 \times 10^{-1}$ & $1.363 \times 10^{-5}$ & $1.191 \times 10^{0}$ & $9.811 \times 10^{4}$ & $5 \cdot 851$ & $1.073 \times 10^{7}$ & $5.84 \times 10^{-4}$ \\
\hline $4.64 \times 10^{-1}$ & $1.363 \times 10^{-5}$ & $1.191 \times 10^{0}$ & $2.113 \times 10^{4}$ & $5 \cdot 851$ & $1.073 \times 10^{7}$ & $2.71 \times 10^{-3}$ \\
\hline $2.15 \times 10^{0}$ & $1.363 \times 10^{-5}$ & $1.191 \times 10^{0}$ & $4.553 \times 10^{3}$ & $5 \cdot 851$ & $1.073 \times 10^{7}$ & $1.26 \times 10^{-2}$ \\
\hline $1.00 \times 10^{1}$ & $1.363 \times 10^{-5}$ & $1.191 \times 10^{0}$ & $9.811 \times 10^{2}$ & $5 \cdot 851$ & $1.073 \times 10^{7}$ & $5.84 \times 10^{-2}$ \\
\hline $4.64 \times 10^{1}$ & $1.351 \times 10^{-5}$ & $1.191 \times 10^{0}$ & $2 \cdot 132 \times 10^{2}$ & $5 \cdot 851$ & $1.063 \times 10^{7}$ & $2.69 \times 10^{-1}$ \\
\hline $2 \cdot 15 \times 10^{2}$ & $1.159 \times 10^{-5}$ & $1.182 \times 10^{0}$ & $5.395 \times 10^{1}$ & $5 \cdot 894$ & $9 \cdot 122 \times 10^{6}$ & $1.06 \times 10^{0}$ \\
\hline $1.00 \times 10^{3}$ & $2.870 \times 10^{-6}$ & $1.143 \times 10^{0}$ & $4.851 \times 10^{1}$ & 6.095 & $2.259 \times 10^{6}$ & $1.18 \times 10^{0}$ \\
\hline $4.64 \times 10^{3}$ & $1.913 \times 10^{-7}$ & $1.133 \times 10^{0}$ & $1.582 \times 10^{2}$ & $6 \cdot 151$ & $1.506 \times 10^{5}$ & $3.62 \times 10^{-1}$ \\
\hline $2.15 \times 10^{4}$ & $3.445 \times 10^{-8}$ & $1.132 \times 10^{0}$ & $1.896 \times 10^{2}$ & $6 \cdot 157$ & $2.711 \times 10^{4}$ & $3.02 \times 10^{-1}$ \\
\hline $1.00 \times 10^{5}$ & $2.704 \times 10^{-8}$ & $1.132 \times 10^{0}$ & $5.200 \times 10^{1}$ & $6 \cdot 154$ & $2.129 \times 10^{4}$ & $1.10 \times 10^{0}$ \\
\hline $4.64 \times 10^{5}$ & $2.673 \times 10^{-8}$ & $1.127 \times 10^{0}$ & $1 \cdot 134 \times 10^{1}$ & $6 \cdot 158$ & $2 \cdot 104 \times 10^{4}$ & $5.04 \times 10^{0}$ \\
\hline $2 \cdot 15 \times 10^{6}$ & $2.671 \times 10^{-8}$ & $1.048 \times 10^{0}$ & $2.445 \times 10^{0}$ & $6 \cdot 156$ & $2 \cdot 102 \times 10^{4}$ & $2.23 \times 10^{1}$ \\
\hline $1.00 \times 10^{7}$ & $2.670 \times 10^{-8}$ & $5.276 \times 10^{-1}$ & $5.268 \times 10^{-1}$ & $6 \cdot 157$ & $2 \cdot 102 \times 10^{4}$ & $6.22 \times 10^{1}$ \\
\hline $4.64 \times 10^{7}$ & $2.671 \times 10^{-8}$ & $1.276 \times 10^{-1}$ & $1.136 \times 10^{-1}$ & $6 \cdot 162$ & $2.102 \times 10^{4}$ & $8.35 \times 10^{1}$ \\
\hline $2 \cdot 15 \times 10^{8}$ & $2.671 \times 10^{-8}$ & $2.767 \times 10^{-2}$ & $2.445 \times 10^{-2}$ & $6 \cdot 158$ & $2 \cdot 102 \times 10^{4}$ & $8 \cdot 86 \times 10^{1}$ \\
\hline $1.00 \times 10^{9}$ & $2.670 \times 10^{-8}$ & $5.963 \times 10^{-3}$ & $5.267 \times 10^{-3}$ & $6 \cdot 156$ & $2 \cdot 102 \times 10^{4}$ & $8.97 \times 10^{1}$ \\
\hline $4.64 \times 10^{9}$ & $2.670 \times 10^{-8}$ & $1.285 \times 10^{-3}$ & $1.135 \times 10^{-3}$ & $6 \cdot 154$ & $2 \cdot 101 \times 10^{4}$ & $8.99 \times 10^{1}$ \\
\hline $2.15 \times 10^{10}$ & $2.671 \times 10^{-8}$ & $2.770 \times 10^{-4}$ & $2.450 \times 10^{-4}$ & $6 \cdot 156$ & $2 \cdot 102 \times 10^{4}$ & $9.00 \times 10^{1}$ \\
\hline $1.00 \times 10^{11}$ & $2 \cdot 670 \times 10^{-8}$ & $5.968 \times 10^{-5}$ & $5 \cdot 268 \times 10^{-5}$ & $6 \cdot 157$ & $2 \cdot 102 \times 10^{4}$ & $9.00 \times 10^{1}$ \\
\hline
\end{tabular}

Table 3. AC components along with its values of polyaniline and its composite using ammonium per sulphate as oxidant in $\mathrm{HCl}$ medium.

\begin{tabular}{|c|c|c|c|c|c|c|}
\hline \multirow[b]{3}{*}{ Sample } & \multicolumn{4}{|c|}{ Electrical properties } & \multirow{3}{*}{$\begin{array}{l}\text { Energy of } \\
\text { act. }(\mathrm{eV})\end{array}$} & \multirow{3}{*}{$\begin{array}{c}\text { Conductivity } \\
\text { at room } \\
\text { temperature } \\
(\mathrm{S} / \mathrm{cm})\end{array}$} \\
\hline & \multicolumn{2}{|c|}{ Grain } & \multicolumn{2}{|c|}{ Electrode } & & \\
\hline & $R(\mathrm{Ohms})$ & $C$ (Farad) & $R(\mathrm{Ohms})$ & $C$ (Farad) & & \\
\hline olyaniline & $4.26 \times 10^{-1}$ & $3.00 \times 10^{-7}$ & $2.93 \times 10^{-2}$ & $1.82 \times 10^{-2}$ & $0 \cdot 058$ & $4.40 \times 10^{-1}$ \\
\hline CdS & $7.06 \times 10^{2}$ & $8 \cdot 15 \times 10^{-10}$ & $2.70 \times 10^{3}$ & $4.19 \times 10^{-7}$ & - & $8.75 \times 10^{-5}$ \\
\hline olyaniline-olyethylene glycol & $3.79 \times 10^{0}$ & $1.11 \times 10^{-9}$ & $2.45 \times 10^{-1}$ & $1.45 \times 10^{-3}$ & 0.064 & $4.28 \times 10^{-2}$ \\
\hline olyaniline-CdS & $1.13 \times 10^{0}$ & $2.67 \times 10^{-8}$ & $5.92 \times 10^{-2}$ & $5.50 \times 10^{-3}$ & 0.021 & $8.75 \times 10^{-5}$ \\
\hline olyaniline-olyethylene glycol-CdS & $9.55 \times 10^{-1}$ & $2.59 \times 10^{-7}$ & - & - & 0.033 & $6 \cdot 12 \times 10^{-2}$ \\
\hline
\end{tabular}

conductivities calculated from the grain resistances match very well with the values for different samples. The relaxation times determined from respective $\mathrm{R} \& \mathrm{C}$ values lie in the range of $1.28 \times 10^{-7}$ to $1.30 \times 10^{-8} \mathrm{~s}$ for grain and $5 \cdot 32-2.51 \times 10^{-4} \mathrm{~s}$ for electrode processes. In all the systems, conductivity increased with temperature indicating the semiconductor behaviour of materials. Some Arrhenius plots are shown in figure 8. The thermal energy of activation determined from the temperature dependence of electrical conductivity is included in table 3 . The values lie in the range of $0.021-0.064 \mathrm{eV}$ which are typical values for organic semiconductors. The regression analysis graph of PAni-PEG-CdS is shown in figure 9. The regression analysis of conductance data as a function of temperature showed three-dimensional variable range hopping charge transport in these materials except for PAni-CdS system in which two dimensional variable-ranges hopping was observed.

\section{Conclusions}

A simple and elegant method for the preparation of conducting hybrid organic-inorganic nanomaterials has been reported. A mechanism has been suggested for the formation of a core-shell nanostructure in the triple hybrid system. The role of water soluble organic polymer; polyethylene glycol as biomimetic synthetic aid has been outlined. The proof of formation of nanosized hybrids have been given from spectral measurements. These materials may be useful for light-harvesting by organic-inorganic semiconductors for solar cell applications because of good absorption both in UV and visible region as well as 
good conductivity for facile charge transfer of photogenerated charge carriers. These aspects are under investigation.

\section{References}

Castelvetro V and Vita C D 2004 Adv. Colloid Interface Sci. 108-109 167

Chandrakanthi R L N and Careem M A 2002 Thin Solid Films 41751

Dallas E, Kallitsis J, Sakkopoulos S, Vitoratas E and Koutsoukos P G 1991 J. Colloid Interface Sci. 141137

Dutta K, De S and De S K 2007 J. Appl. Phys. 101093711

Godovsky D Y, Varfolomeev A E, Zaretsky D F, Chandrakanthi R L N, Kundig A, Weder C and Caseri W 2001 J. Mater. Chem. 112465

Gangopadhyay R, De A and Ghosh G 2001 Synth. Metals 123 21

Jang J, Ha J and Lim B 2006 Chem. Comm. 1622

Klem B M T, Young M and Douglas T 2005 Materials Today 8 28

Kallitsis J, Koumanakos E, Dallas E, Sakkopoulos S and Koutsoukos P G 1989 J. Chem. Soc. Chem. Comm. 1146

Khiew P S, Huang N M, Radiman S and Soot-Ahmad Md 2004 Mater. Lett. 58516

Khanna P K, Lonkar S P, Subbarao V V V S and Jun K-W 2004 Mater. Chem. Phys. 8749
Khanna P K, Gokhale R R, Subbarao V V V S, Singh N, Jun K -W and Das B K 2005 Mater. Chem. Phys. 94454

Khanna P K, Kulkarni M V, Singh N, Lonkar S P, Subbarao V V V S and Viswanath A K 2006 Mater. Chem. Phys. 9524

Kang Y and Kim D 2006 Solar Energy Mater. Solar Cells 90166

Kang E T, Neoh K G and Tan K L 1998 Prog. Polym. Sci. 23277

Mann A S (ed.) 1997 Biomimetic materials chemistry (Wiley)

Ma X, Lu G and Yang B 2002 Appl. Surf. Sci. 187235

Nalwa H S (ed.) 2001 Nanostructured materials and nanotechnology (Academic Press)

Nalwa H S (ed.) 2002 Magnetic nanostructures (New York: American Science Publishers)

Singh N, Kulkarni M V, Lonkar S P, Viswanath A K and Khanna P K 2007 Synth. React. Inorg., Metal-Organic Nanometal Chem. 37153

Pethakar S, Patil R C, Kher J A and Vijayamohanan K 1999 Thin Solid Films 349105

Pouget J P, Jozefowicz M E, Epstein A J, Tang X and MacDiarmid A G 1991 Macromolecules 24779

Trivedi D C 1997 Handbook of conductive molecules and polymers II. conductive polymers: synthesis and electrical properties (ed.) H S Nalwa (New York: John Wiley)

Yang L, Shen Q, Zhou J and Jiang K 2006 Mater. Chem. Phys. 98125

Yonema H, Tokuda M and Kuwabata S 1994 Electrochim. Acta 391315

Zang Q, Atay T, Tisshler J R, Bardley M S, Bulovic V and Nurmikko A A 2007 Nature Nanotech. 2555 